\title{
DESEMPENHO AGRONÔMICO DE CULTIVARES DE SOJA EM DIFERENTES REGIÕES DO ESTADO DE SÃO PAULO, SAFRA 2018/19
}

\author{
SANTOS, Guilherme Xavier Lucio dos ${ }^{1}$; BÁRBARO-TORNELI, Ivana Marino ${ }^{2}$; MARTINS, \\ Monica Helena ${ }^{1}$; CORDEIRO JUNIOR, Paulo Sérgio ${ }^{3}$; POLLI, Katia Cristina ${ }^{4}$; CORREIA, Alessandra \\ Neves ${ }^{1}$; FINOTO, Everton Luis ${ }^{5}$; BARROS, Vera Lúcia Nishijima Paes de ${ }^{6}$; GALLO, Paulo Boller ${ }^{7}$ \\ SOARES, Maria Beatriz Bernardes ${ }^{8}$; DONA, Sérgio ${ }^{9}$; NAKAYAMA, Fernando Takayuki ${ }^{10}$; SÁ, Luís \\ Antônio Dias de ${ }^{11} ;$ LEÃ̃O, Paulo César da Luz ${ }^{12}$
}

ISSUE DOI: $10.3738 / 1982.2278 .3629$

\begin{abstract}
RESUMO: Para a obtenção de elevadas produtividades de soja é fundamental a escolha da cultivar e da época de semeadura mais adequadas para cada região de produção. Nessa pesquisa, objetivou-se estudar o desempenho agronômico de diferentes cultivares de soja nos municípios de Capão Bonito, Pindorama, Mococa, Mogi Mirim e Tanabi, no Estado de São Paulo, no ano-agrícola de 2018/19. O delineamento experimental utilizado foi de blocos casualizados, com diferentes variedades nas localidades e 3 repetições. As médias foram agrupadas pelo teste de Scott-Knott a $5 \%$ de probabilidade. As características avaliadas foram: altura de plantas, altura de inserção da primeira vagem, população de plantas e produtividade de grãos. Há cultivares comerciais que apresentam tolerância às condições adversas de cada região apresentando produtividades superiores à média estadual.
\end{abstract}

Palavras Chave: Glycine Max (L.) Merrill. Produtividade de Grãos. Ambiente de produção.

\section{AGRONOMIC PERFORMANCE OF SOYBEAN CULTIVARS IN THE VALLEY OF, SEASON 2018/19}

\begin{abstract}
SUMMARY: In order to obtain high soybean yields, it is essential to choose the most suitable cultivar and sowing season for each production region. The objective of this research was to study the agronomic performance of different soybean cultivars in the municipalities of Capão Bonito, Pindorama, Mococa, Mogi Mirim and Tanabi, in the state of São Paulo, in the agricultural year of 2018/19. The experimental design was of randomized blocks, with different varieties in the localities and 3 replicates. The means were grouped by the Scott-Knott test at $5 \%$ probability. The evaluated characteristics were: plant height, first pod insertion height, plant population and grain yield. There are commercial cultivars that present tolerance to the adverse conditions of each region presenting yields higher than the state average.
\end{abstract}

Keywords: Glycine Max (L.) Merrill. Grains Yield. Producing Environment.

\footnotetext{
${ }^{1}$ Eng. Agrônomo, Bolsista de Aperfeiçoamento Técnico, FUNDAG/Agência Paulista de Tecnologia dos Agronegócios (APTA)/Sec. de Agricultura e Abastecimento (SAA) - Polo Regional Centro Norte - Pindorama,SP;

${ }^{2}$ Eng. Agrônoma, Dra. Pesquisadora Científica, APTA/SAA - Polo Regional Alta Mogiana - Colina, SP;

${ }^{3}$ Eng. Agrônomo, Mestrando em Produção Vegetal, FCAV-UNESP- Jaboticabal,SP;

${ }^{4}$ Graduando em Agronomia, Centro Universitário de Rio Preto-UNIRP, Bolsista PIBIC/CNPq/APTA/SAA - Polo Regional Centro Norte - Pindorama;

${ }^{5}$ Eng. Agrônomo, Dr., Pesquisador Cientifico, APTA/SAA - Polo Regional Centro Norte - Pindorama;

${ }^{6}$ Eng. Agrônoma, Msc., Pesquisadora Cientifica, APTA/SAA - IAC/UPD Capão Bonito;

${ }^{7}$ Eng. Agrônomo,.Msc., Pesquisador Cientifico, APTA/SAA - IAC/UPD Mococa;

${ }^{8}$ Eng. Agrônoma, Msc., Pesquisadora Cientifica, APTA/SAA - Polo Regional Centro Norte - Pindorama;

${ }^{9}$ Eng. Agrônomo, Msc., Pesquisador Cientifico, APTA/SAA - Polo Regional Médio Paranapanema - Assis;

${ }^{10}$ Eng. Agrônomo, Dr., Pesquisador Cientifico, APTA/SAA - Polo Regional Alta Paulista - Adamantina;

${ }^{11}$ Engenheiro Agrônomo,Assistente Agropecuário - CDRS - EDR,CA Mogi Mirim;

${ }^{12}$ Engenheiro Agrônomo,Assistente Agropecuário - CDRS - EDR,CA Orlândia.
} 


\section{INTRODUÇÃO}

A cultura da soja [Glycine max (L.) Merrill] possui grande relevância para a agricultura e economia mundial. A produção, em 2018, atingiu a 369,32 milhões de toneladas, sendo os Estados Unidos e o Brasil, primeiro e segundo maiores produtores respectivamente (USDA, 2018). Na safra 2017/18, o Brasil produziu 115,40 milhões de toneladas com uma produtividade média de $3.394 \mathrm{~kg} \mathrm{ha}^{-1}$ (CONAB, 2019). A soja é responsável pela formação de uma complexa estrutura de produção, armazenamento, processamento e de comercialização em todos os países onde é cultivada em larga escala e, a grande demanda no mercado internacional, proporcionou rápida expansão dessa cultura no Brasil, que ocorreu pela tomada de áreas cultivadas com outras culturas e, também, pela conquista de novas fronteiras agrícolas (REZENDE; CARVALHO 2007).

A soja vem apresentando rápido avanço no estado de São Paulo nos últimos dez anos, graças a parcerias com o setor da cana-de-açúcar, na renovação das lavouras. Na safra 2008/09, o grão ocupava 531,3 mil hectares no estado, segundo dados da Companhia Nacional de Abastecimento (CONAB). Na safra 2018/19, a estimativa é passar pela primeira vez de 1 milhão de hectares, atingindo 1,026 milhão, quase o dobro (CONAB, 2019).

Estudos de competição de cultivares são de extrema importância para a recomendação para determinada região, pois, materiais mais adaptados oferecem maiores níveis de produtividade e, o sucesso em relação à produtividade é condicionado pelo genótipo do material e pela sua interação com as variações ambientais (YUYAMA, 1991).

Segundo Peixoto et al. (2000), existe grande variabilidade de comportamento entre cultivares de soja com relação a sensibilidade à época e local de semeadura, as quais se encontram disponíveis no mercado apresentando grande diversificação, principalmente quanto à interação genótipo x ambiente e, sendo por isso, desejável que os produtores tenham conhecimentos mais aprofundados destes cultivares nos diferentes ambientes.

Diante desse contexto, objetivou-se avaliar o desempenho agronômico de cultivares comerciais de soja, em diferentes condições edafoclimáticas, em cinco locais do Estado de São Paulo, inseridos nas Regiões 203 e 302.

\section{MATERIAL E MÉTODO}

Na safra 2018/19 os ensaios para avaliação do desempenho agronômico de cultivares de soja foram implantados em Unidades de Pesquisa e Desenvolvimento (UPD) da Agência Paulista de Tecnologia dos Agronegócios (APTA), órgão estadual da Secretaria de Agricultura e Abastecimento (SAA) e, em propriedades particulares nos seguintes municípios: Capão Bonito (UPD - APTA/IAC), Mococa (UPD - APTA/IAC), Mogi Mirim (propriedade particular), Pindorama (Apta Regional Centro Norte) e Tanabi (propriedade particular). Utilizou-se o delineamento estatístico de blocos casualizados com três repetições. As parcelas experimentais foram constituídas de quatro linhas de 5,0 m de comprimento, espaçadas de $0,5 \mathrm{~m}$, considerando-se para as avaliações agronômicas apenas as duas linhas centrais.

Dados meteorológicos de cada local ao longo da execução do experimento, período 10/10/2018 a 15/04/2019, obtidos por meio do Centro Integrado de Informações Agrometeorológicas (CIIAGRO), estão representados a seguir (Figuras 1, 2, 3, 4 e 5). 
Figura 1. Dados de precipitação, temperatura máxima e mínima, Capão Bonito-SP.

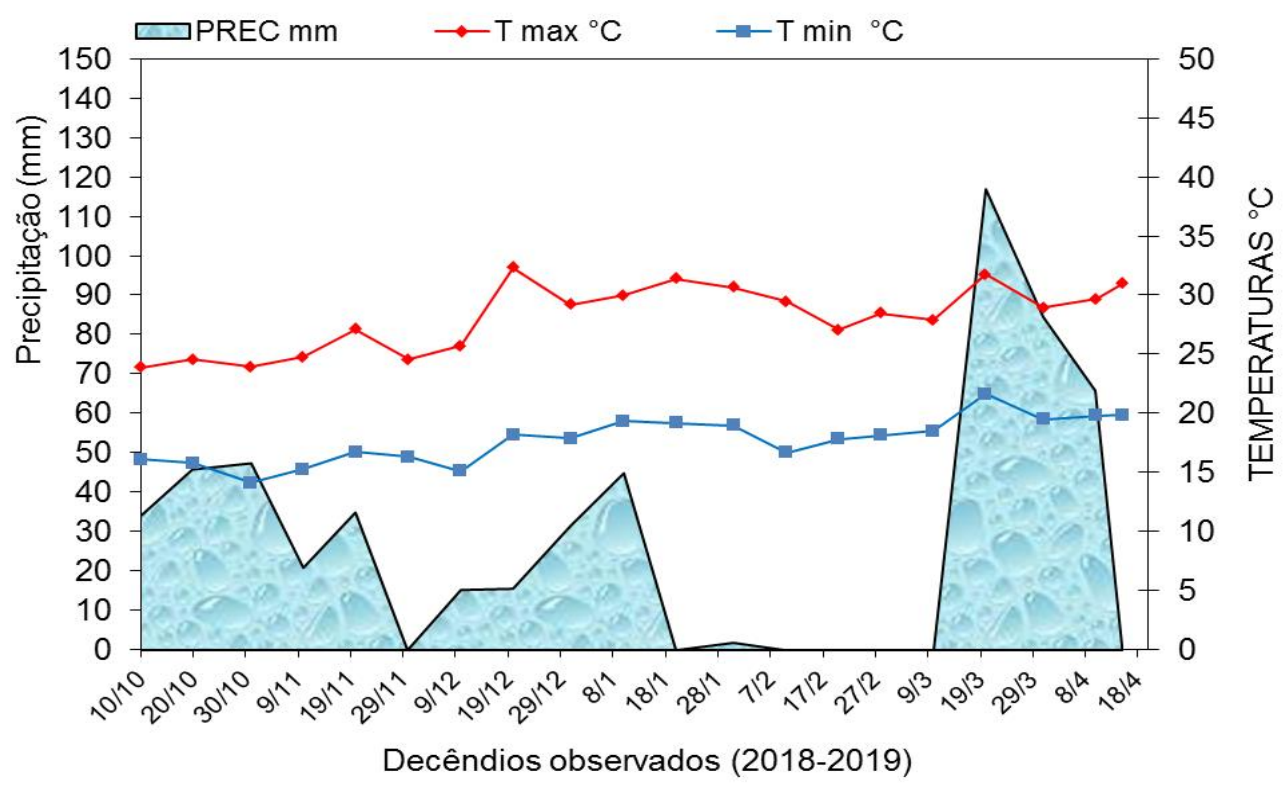

Figura 2. Dados de precipitação, temperatura máxima e mínima registrados, Pindorama-SP.

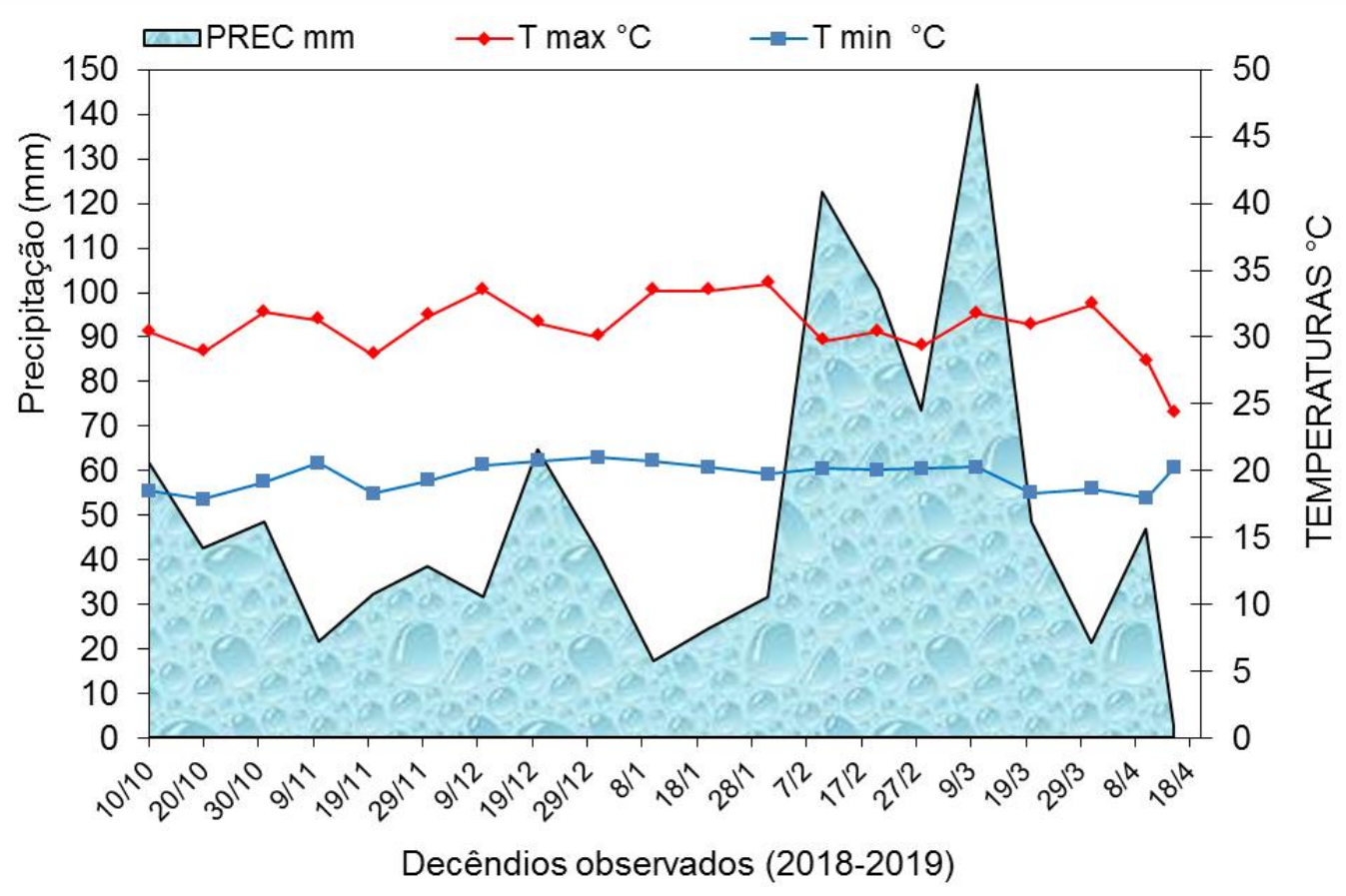


Figura 3. Dados de precipitação, temperatura máxima e mínima registrados em Mococa-SP.

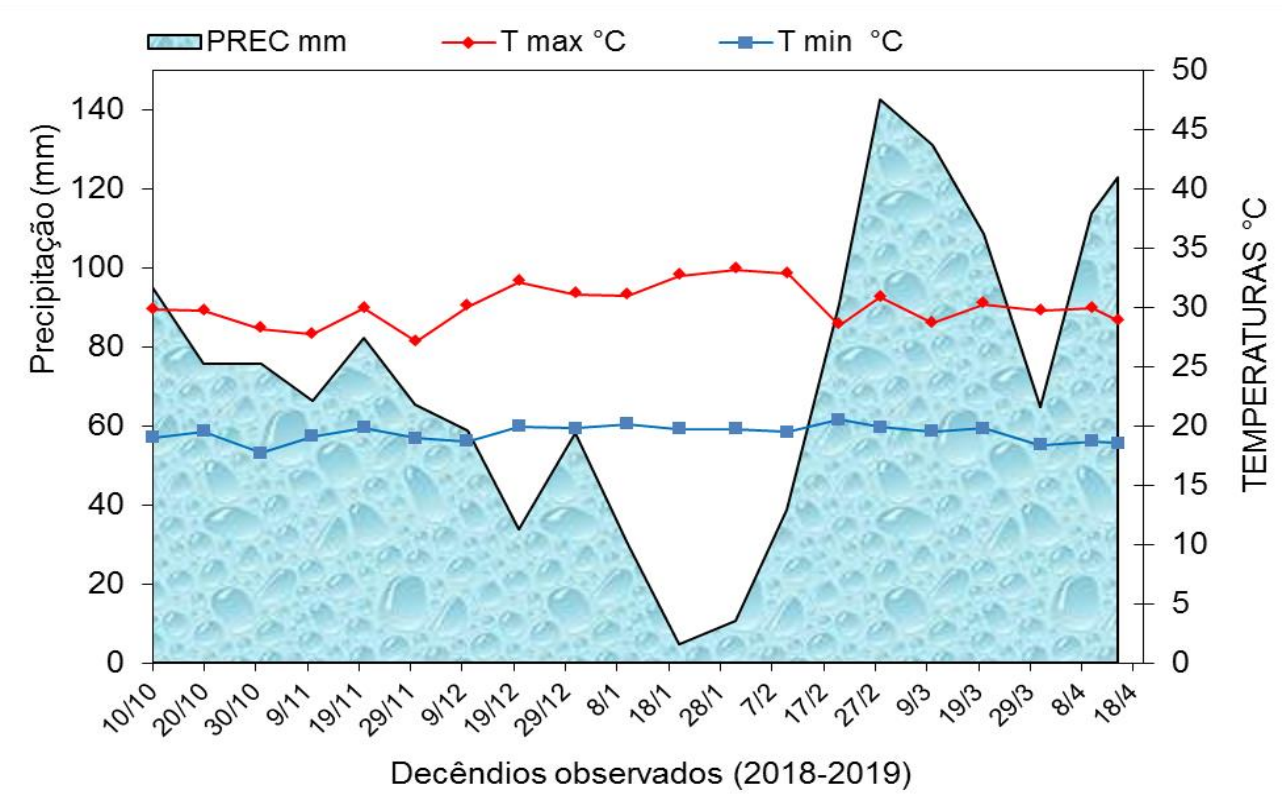

Figura 4. Dados de precipitação, temperatura máxima e mínima registrados, Mogi Mirim-SP.

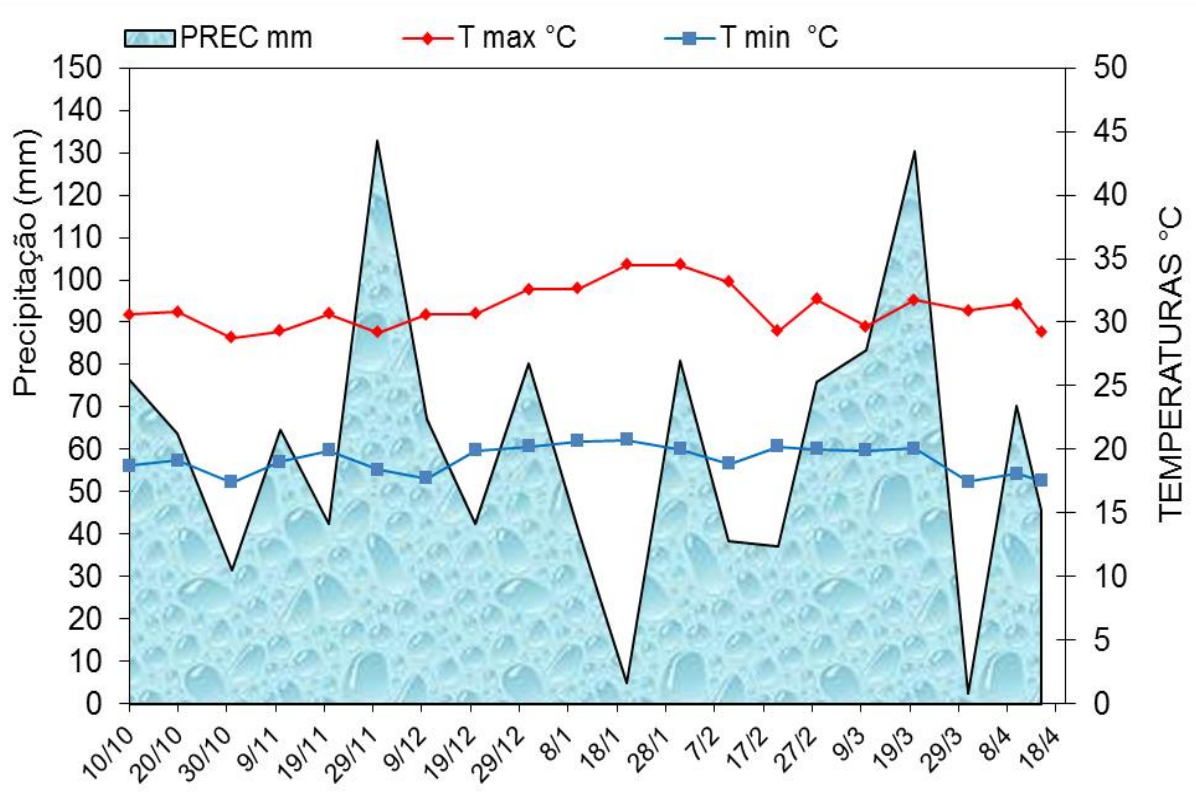

Decêndios observados (2018-2019) 
Figura 5. Dados de precipitação, temperatura máxima e mínima registrados, em Tanabi-SP.

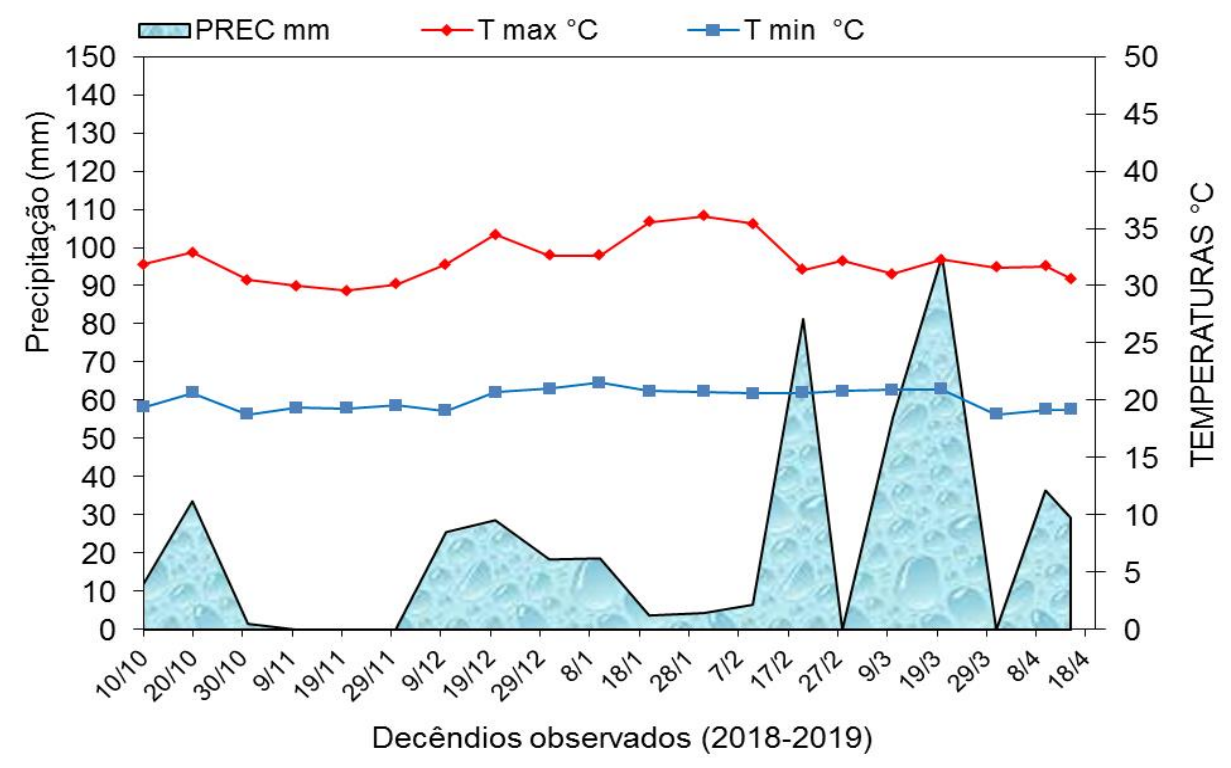

No Quadro 1 estão representadas as características: tipo de solo, altitude e plantio de cada local.

Quadro 1. Caracterização dos locais onde foram instalados os experimentos, safra 2018/19.

\begin{tabular}{|c|c|c|c|}
\hline Município & Altitude & Região edafoclimática & Data de semeadura \\
\hline Capão Bonito-SP & $730 \mathrm{~m}$ & 203 & $22 / 11 / 2018$ \\
\hline Mococa-SP & $665 \mathrm{~m}$ & 302 & $27 / 11 / 2018$ \\
\hline Mogi Mirim-SP & $611 \mathrm{~m}$ & 203 & $14 / 11 / 2018$ \\
\hline Pindorama-SP & $527 \mathrm{~m}$ & 302 & $05 / 11 / 2018$ \\
\hline Tanabi-SP & $518 \mathrm{~m}$ & 302 & $01 / 11 / 2018$ \\
\hline
\end{tabular}

Em cada experimento, foram avaliadas de 29 a 35 cultivares de soja, indicadas para cada local (Quadro 2).

Quadro 2. Relação de cultivares avaliadas nos municípios paulistas de Capão Bonito, Mococa, Mogi Mirim, Pindorama e Tanabi, safra 2018/19.

(Continua)

\begin{tabular}{|c|c|c|c|c|c|c|}
\hline Empresa & Cultivares & $\begin{array}{c}\text { Capão } \\
\text { Bonito }\end{array}$ & Pindorama & Mococa & $\begin{array}{c}\text { Mogi } \\
\text { Mirim }\end{array}$ & Tanabi \\
\hline AGROESTE & AS 3590 & X & & X & X & \\
\hline AGROESTE & AS 3610 & & & X & & \\
\hline AGROESTE & AS 3680 & X & X & X & X & \\
\hline AGROESTE & AS 3730 & X & & $X$ & $X$ & $X$ \\
\hline BRASMAX & BMX 63 I 64 & X & X & & X & \\
\hline BRASMAX & BMX 71b66 & & & & & $X$ \\
\hline BRASMAX & BMX DESAFIO & & & & & $X$ \\
\hline BRASMAX & BMX FOCO & & & & & $X$ \\
\hline
\end{tabular}


Quadro 2. Relação de cultivares avaliadas nos municípios paulistas de Capão Bonito, Mococa, Mogi Mirim, Pindorama e Tanabi, safra 2018/19.

(Continuação)

\begin{tabular}{|c|c|c|c|c|c|c|}
\hline Empresa & Cultivares & $\begin{array}{l}\text { Capão } \\
\text { Bonito }\end{array}$ & Pindorama & Mococa & $\begin{array}{c}\text { Mogi } \\
\text { Mirim } \\
\end{array}$ & Tanabi \\
\hline BRASMAX & BMX ÚNICA & & & & & $X$ \\
\hline EMBRAPA & BRS 1001 & $X$ & & $X$ & $X$ & \\
\hline EMBRAPA & BRS 1003 & $X$ & $X$ & & $\mathrm{X}$ & \\
\hline EMBRAPA & BRS 1010 & $X$ & $X$ & & $X$ & \\
\hline EMBRAPA & BRS 1074 & & $X$ & $X$ & & \\
\hline EMBRAPA & BRS 388 & $X$ & $X$ & $\mathrm{X}$ & $X$ & \\
\hline EMBRAPA & BRS 399 & $X$ & $X$ & & $X$ & \\
\hline EMBRAPA & BRS 413 & $X$ & $X$ & & $X$ & \\
\hline EMBRAPA & BRS 433 & & $X$ & & & \\
\hline EMBRAPA & BRS 7380 & $X$ & $X$ & $\mathrm{X}$ & $X$ & $X$ \\
\hline BAYER & BS 2606 & $X$ & & & $X$ & \\
\hline COODETEC & CD 2700 & & & & & $X$ \\
\hline DONMARIO & DM 68169 & & & & & $X$ \\
\hline FT Sementes & FTR 4160 & $X$ & $X$ & & $X$ & \\
\hline FT Sementes & FTR ANTARI & & & & & $X$ \\
\hline LG Sementes & LG 11 & & & & & $X$ \\
\hline LG Sementes & LG 158 & & & $X$ & & \\
\hline LG Sementes & LG 162 & & & $X$ & & \\
\hline LG Sementes & LG 179 & & & $X$ & & \\
\hline LG Sementes & LG 19 & & & & & $X$ \\
\hline LG Sementes & LG 54 & & & & & $X$ \\
\hline LG Sementes & LG 60i63 & & & & & $X$ \\
\hline LG Sementes & LG 79 & & & & & $X$ \\
\hline MONSOY & M 5917 & $X$ & $X$ & $X$ & $\mathrm{X}$ & \\
\hline MONSOY & M 5947 & $X$ & $X$ & $X$ & $X$ & \\
\hline MONSOY & M 6210 & $X$ & $\mathrm{X}$ & $X$ & & \\
\hline MONSOY & M 6410 & $X$ & $\mathrm{X}$ & $X$ & $X$ & $X$ \\
\hline MONSOY & M 7110 & & & & & $\mathrm{X}$ \\
\hline MONSOY & M 7739 & & & & & $X$ \\
\hline NIDERA & NA 5909 & & & & & $X$ \\
\hline NIDERA & NA 7337 & & & & & $X$ \\
\hline NIDERA & NS 6700 & $X$ & $X$ & $X$ & $X$ & \\
\hline NIDERA & NS 6906 & $X$ & & $X$ & $X$ & \\
\hline NIDERA & NS 6990 & $X$ & $X$ & $X$ & $X$ & \\
\hline NIDERA & NS 7007 & & $X$ & & & $X$ \\
\hline NIDERA & NS 7202 & & & & & $X$ \\
\hline NIDERA & NS 7767 & & $X$ & & & \\
\hline PIONEER & P9 R10 & & & & & $X$ \\
\hline
\end{tabular}


Quadro 2. Relação de cultivares avaliadas nos municípios paulistas de Capão Bonito, Mococa, Mogi Mirim, Pindorama e Tanabi, safra 2018/19.

(Conclusão)

\begin{tabular}{|c|c|c|c|c|c|c|}
\hline Empresa & Cultivares & $\begin{array}{c}\text { Capão } \\
\text { Bonito }\end{array}$ & Pindorama & Mococa & $\begin{array}{c}\text { Mogi } \\
\text { Mirim }\end{array}$ & Tanabi \\
\hline PIONEER & P95 R 90 & & & & & $\mathrm{X}$ \\
\hline PIONEER & P96 R20 & & & & & $\mathrm{X}$ \\
\hline PIONEER & P96 R70 & & & & & $\mathrm{X}$ \\
\hline PIONEER & P97 R22 & & & & & $\mathrm{X}$ \\
\hline PIONEER & P97 R50 & & & & & $\mathrm{X}$ \\
\hline SEEDCORP & PARANAIBA & & & & & $\mathrm{X}$ \\
\hline KWS & RK 6316 & $\mathrm{X}$ & $\mathrm{X}$ & $\mathrm{X}$ & $\mathrm{X}$ & \\
\hline KWS & RK 6813 & $\mathrm{X}$ & $\mathrm{X}$ & $\mathrm{X}$ & $\mathrm{X}$ & \\
\hline KWS & RK 7518 & $\mathrm{X}$ & $\mathrm{X}$ & $\mathrm{X}$ & $\mathrm{X}$ & \\
\hline SYNGENTA & SYN 13610 & $\mathrm{X}$ & $\mathrm{X}$ & $\mathrm{X}$ & $\mathrm{X}$ & $\mathrm{X}$ \\
\hline SYNGENTA & SYN 1366 & $\mathrm{X}$ & $\mathrm{X}$ & $\mathrm{X}$ & $\mathrm{X}$ & \\
\hline SYNGENTA & SYN 15640 & $\mathrm{X}$ & $\mathrm{X}$ & $\mathrm{X}$ & $\mathrm{X}$ & $\mathrm{X}$ \\
\hline BASF & TEC 7548 & & $\mathrm{X}$ & & & \\
\hline TMG & TMG 7061 & & & & & $\mathrm{X}$ \\
\hline TMG & TMG 7062 & $\mathrm{X}$ & $\mathrm{X}$ & $\mathrm{X}$ & $\mathrm{X}$ & $\mathrm{X}$ \\
\hline TMG & TMG 7063 & $\mathrm{X}$ & $\mathrm{X}$ & $\mathrm{X}$ & $\mathrm{X}$ & $\mathrm{X}$ \\
\hline TMG & TMG 7067 & & & $\mathrm{X}$ & & $\mathrm{X}$ \\
\hline EMBRAPA & VALIOSA & & $\mathrm{X}$ & $\mathrm{X}$ & $\mathrm{X}$ & $\mathrm{X}$ \\
\hline BASF & W791 & & $\mathrm{X}$ & & & \\
\hline
\end{tabular}

A adubação de plantio foi realizada seguindo recomendação baseada no resultado da análise de solo de cada local. O controle de plantas daninhas, pragas e doenças ocorreram conforme recomendações técnicas preconizadas para a cultura na região.

Foram avaliados os caracteres: população final de plantas, obtida pela contagem das plantas presentes em dois metros de linha, em todas as parcelas experimentais; altura de plantas (APM) e da inserção da primeira vagem (AIV), definida pela medição de 4 plantas por parcela por ocasião da colheita; e produtividade de grãos (PG), determinada através da colheita das duas linhas centrais de 5 metros, em cada parcela experimental. A umidade dos grãos foi determinada e os dados de produtividade foram corrigidos para $13 \%$ de umidade.

Análises de variância individuais de cada local foram devidamente efetuadas e as médias foram agrupadas pelo Teste de Scott-Knott a 5\% de probabilidade pelo software AgroEstat (BARBOSA; MALDONADO, 2015).

\section{RESULTADO E DISCUSSÃO}

As características agronômicas da cultura da soja, em Capão Bonito, Pindorama, Mococa, Mogi Mirim e Tanabi estão demonstradas nas Tabelas 1, 2, 3, 4 e 5. Na safra 2018/19, as cultivares avaliadas apresentaram produtividades de grãos variando de 1.127,00 à 5.161,33 $\mathrm{kg} \mathrm{ha}^{-1}$. Sendo que, em todos os locais avaliados houve diferença significativa em relação à altura das plantas na maturação, altura de 
inserção da primeira vagem e produtividade de grãos, confirmando que os genótipos apresentam características agronômicas bastante diferentes, de acordo com Cruz et al. (2010)

Os ensaios realizados em Capão Bonito e Mogi Mirim apresentaram as maiores médias de produtividade com 4.493,22 e 3.902,95 $\mathrm{kg} \mathrm{ha}^{-1}$, respectivamente. Em Pindorama, Tanabi e Mococa foram obtidas médias de produtividades menores 3.209,72, 1.851,81 e 1.879,17 $\mathrm{kg} \mathrm{ha}^{-1}$, respectivamente. Lembrando que, os valores de Capão Bonito e Mogi Mirim foram superiores a média obtida no estado de São Paulo (3.424 kg ha-1), na safra anterior (CONAB, 2017).

O potencial de rendimento de grãos está relacionado diretamente às condições edafoclimáticas, nutricionais, livre da ação de pragas e doenças e com os demais estresses efetivamente controlados segundo Evans (1993). Assim, observa-se nas figuras 1 e 4 que nas regiões de Capão Bonito e Mogi Mirim ocorreram melhores condições de chuva e temperatura ideal para a planta.

Tabela 1. Média dos caracteres agronômicos obtidos de ensaio comparativo de 28 cultivares de soja, conduzido em Capão Bonito, SP. Safra 2018/19.

(Continua)

\begin{tabular}{|c|c|c|c|c|}
\hline Cultivar & APM & AIV & POP & PG \\
\hline RK 6316 & $101,67 \mathrm{a}$ & $10,00 \mathrm{~d}$ & $217333,33 \mathrm{~g}$ & $5054,00 \mathrm{a}$ \\
\hline TMG 7062 & 106,67 a & $20,00 \mathrm{a}$ & $212000,00 \mathrm{~g}$ & 5036,67 a \\
\hline SYN 15640 & $105,00 \mathrm{a}$ & $15,00 \mathrm{c}$ & $234666,67 \mathrm{f}$ & 4958,67 a \\
\hline BMX 63 I 64 & $105,00 \mathrm{a}$ & $20,00 \mathrm{a}$ & 330666,67 a & 4929,33 a \\
\hline AS 3590 & 96,67 a & $15,00 \mathrm{c}$ & $215333,33 \mathrm{~g}$ & $4826,67 \mathrm{a}$ \\
\hline BS 2606 & $85,00 \mathrm{~b}$ & $15,00 \mathrm{c}$ & $268666,67 \mathrm{~d}$ & $4793,00 \mathrm{~b}$ \\
\hline SYN 13610 & 103,33 a & $15,00 \mathrm{c}$ & $312666,67 \mathrm{~b}$ & $4735,00 \mathrm{~b}$ \\
\hline TMG 7063 & 95,00 a & $20,00 \mathrm{a}$ & $332000,00 \mathrm{a}$ & $4722,67 \mathrm{~b}$ \\
\hline M 6210 & $105,00 \mathrm{a}$ & $15,00 \mathrm{c}$ & 335333,33 a & $4667,67 \mathrm{~b}$ \\
\hline BRS 388 & $90,00 \mathrm{~b}$ & $10,00 \mathrm{~d}$ & $311333,33 \mathrm{~b}$ & $4539,00 \mathrm{c}$ \\
\hline BRS 413 & $101,67 \mathrm{a}$ & $18,33 \mathrm{~b}$ & $196666,67 \mathrm{~h}$ & $4538,67 \mathrm{c}$ \\
\hline RK 6813 & $100,00 \mathrm{a}$ & $10,00 \mathrm{~d}$ & $215333,33 \mathrm{~g}$ & $4510,33 \mathrm{c}$ \\
\hline M 5917 & $100,00 \mathrm{a}$ & $5,00 \mathrm{e}$ & $274666,67 d$ & $4493,67 \mathrm{c}$ \\
\hline SYN 1366 & 96,67 a & $15,00 \mathrm{c}$ & $274000,00 \mathrm{~d}$ & $4473,67 \mathrm{c}$ \\
\hline AS 3680 & $95,00 \mathrm{a}$ & $15,00 \mathrm{c}$ & $264666,67 \mathrm{~d}$ & $4438,67 \mathrm{c}$ \\
\hline BRS 399 & 98,33 a & $15,00 \mathrm{c}$ & $270000,00 \mathrm{~d}$ & $4363,67 \mathrm{~d}$ \\
\hline FTR 4160 & 96,67 a & $15,00 \mathrm{c}$ & $237333,33 \mathrm{f}$ & $4362,00 \mathrm{~d}$ \\
\hline NS 6700 & $96,67 \mathrm{a}$ & $18,33 \mathrm{~b}$ & $212666,67 \mathrm{~g}$ & $4357,00 \mathrm{~d}$ \\
\hline BRS 1003 & $90,00 \mathrm{~b}$ & $10,00 \mathrm{~d}$ & $262666,67 \mathrm{~d}$ & $4353,67 \mathrm{~d}$ \\
\hline BRS 1010 & 96,67 a & $10,00 \mathrm{~d}$ & $236000,00 \mathrm{f}$ & $4311,67 \mathrm{~d}$ \\
\hline NS 6990 & $76,67 \mathrm{~b}$ & $10,00 \mathrm{~d}$ & 328666,67 a & $4303,67 \mathrm{~d}$ \\
\hline M 6410 & $86,66 \mathrm{~b}$ & $15,00 \mathrm{c}$ & $290666,67 \mathrm{c}$ & $4257,33 \mathrm{e}$ \\
\hline NS 6906 & $105,00 \mathrm{a}$ & $20,00 \mathrm{a}$ & $214666,67 \mathrm{~g}$ & $4244,33 \mathrm{e}$ \\
\hline BRS 1001 & $106,67 \mathrm{a}$ & $20,00 \mathrm{a}$ & $254000,00 \mathrm{e}$ & $4235,00 \mathrm{e}$ \\
\hline
\end{tabular}


Tabela 1. Média dos caracteres agronômicos obtidos de ensaio comparativo de 28 cultivares de soja, conduzido em Capão Bonito, SP. Safra 2018/19.

(Conclusão)

\begin{tabular}{ccccc}
\hline Cultivar & APM & AIV & POP & PG \\
BRS 7380 & $93,33 \mathrm{a}$ & $5,00 \mathrm{e}$ & $269333,33 \mathrm{~d}$ & $4143,67 \mathrm{f}$ \\
M 5947 & $100,00 \mathrm{a}$ & $15,00 \mathrm{c}$ & $234666,67 \mathrm{f}$ & $4086,33 \mathrm{f}$ \\
AS 3730 & $96,67 \mathrm{a}$ & $10,00 \mathrm{~d}$ & $216000,00 \mathrm{~g}$ & $4083,67 \mathrm{f}$ \\
RK 7518 & $95,00 \mathrm{a}$ & $15,00 \mathrm{c}$ & $236000,00 \mathrm{f}$ & $3990,67 \mathrm{f}$ \\
\hline Média & 97,32 & 14,17 & $259.214,29$ & 4493,22 \\
\hline CV\% & 6,83 & 5,34 & 2,24 & 2,50 \\
\hline F & $3,37^{* *}$ & $97,46^{* *}$ & $161,34^{* *}$ & $21,11^{* *}$
\end{tabular}

Médias de três repetições seguidas de mesmas letras minúsculas na coluna não diferem estatisticamente pelo teste de Scott Knott a $5 \%$ de probabilidade. APM = altura de planta na maturação em $\mathrm{cm}$; AIV = altura de inserção da primeira vagem em $\mathrm{cm}$; $\mathrm{POP}=$ população de plantas em plantas ha ${ }^{-1} \mathrm{e} \mathrm{PG}=$ produtividade de grãos em $\mathrm{kg} \mathrm{ha}^{-1}{ }^{3}$.

Destacou-se em Capão Bonito e Mogi Mirim, a cultivar RK6316, com média entre estes de $5.052,16 \mathrm{~kg} \mathrm{ha}^{-1}$. Em Pindorama, a cultivar VALIOSA, que apresentou produtividade de grãos de 5.161,33 $\mathrm{kg} \mathrm{h}^{-1}$, formando com a W791 o grupo das mais produtivas, destacando-se das demais. Em Mococa, a mais produtiva foi a cultivar AS 3590 alcançando a produtividade de $2.916,67 \mathrm{~kg} \mathrm{ha}^{-1}$ juntamente com a AS 3680. Já em Tanabi, a cultivar SYN 15640 alcançou 2.894,00 $\mathrm{kg} \mathrm{ha}^{-1}$ formando com a P96 R70 o grupo das mais produtivas.

Com relação às variáveis altura de planta e inserção de vagens na maturação, observa-se que a média das cultivares, apresentaram valores consideráveis para a colheita mecanizada, uma vez que os valores estão acima dos padrões exigidos (BONETTI, 1983).

Borges et al., (2018), observaram que a cultivar AS 3730 apresentou elevada produtividade (5156 $\mathrm{kg} \mathrm{ha}{ }^{1}$ ), diferindo das demais cultivares avaliadas em Pindorama, na safra 2017/18, quando ocorreu acentuado índice de chuva na primeira semana de janeiro, proporcionando condições hídricas favoráveis ao desenvolvimento da cultura na fase reprodutiva. Nesta safra, a mesma cultivar não apresentou boa produtividade (tabela 2), mostrando-se não tolerante ao estresse hídrico, observado na figura 3, onde em janeiro, ocorreu baixa precipitação. A cultivar TMG 7063 comparada na safra de 2018/19, teve a sua produtividade semelhante em diferentes condições climáticas, observando-se sua resistência ao déficit hídrico sem interferência na sua produção.

Tabela 2. Média dos caracteres agronômicos obtidos de ensaio comparativo de 30 cultivares de soja, conduzido em Pindorama, SP. Safra 2018/19.

(Continua)

\begin{tabular}{ccccc}
\hline Cultivar & APM & AIV & POP & PG \\
\hline VALIOSA & $80,70 \mathrm{~b}$ & $14,37 \mathrm{a}$ & $413333,33 \mathrm{a}$ & $5161,33 \mathrm{a}$ \\
W791 & $82,27 \mathrm{~b}$ & $15,10 \mathrm{a}$ & $420444,33 \mathrm{a}$ & $4904,00 \mathrm{a}$ \\
NS 7767 & $83,27 \mathrm{a}$ & $14,10 \mathrm{a}$ & $407333,33 \mathrm{a}$ & $4746,33 \mathrm{~b}$ \\
NS 7007 & $87,37 \mathrm{a}$ & $12,00 \mathrm{a}$ & $313333,33 \mathrm{~b}$ & $4504,00 \mathrm{~b}$ \\
TMG 7062 & $81,00 \mathrm{~b}$ & $11,10 \mathrm{a}$ & $218000,00 \mathrm{~b}$ & $4141,33 \mathrm{c}$ \\
TEC 7548 & $96,50 \mathrm{a}$ & $20,27 \mathrm{a}$ & $375666,67 \mathrm{a}$ & $4131,67 \mathrm{c}$
\end{tabular}


Tabela 2. Média dos caracteres agronômicos obtidos de ensaio comparativo de 30 cultivares de soja, conduzido em Pindorama, SP. Safra 2018/19.

(Conclusão)

\begin{tabular}{ccccc}
\hline Cultivar & APM & AIV & POP & PG \\
BRS 1074 & $90,53 \mathrm{a}$ & $12,10 \mathrm{a}$ & $375666,67 \mathrm{a}$ & $3811,00 \mathrm{~d}$ \\
NS 6700 & $86,30 \mathrm{a}$ & $12,43 \mathrm{a}$ & $299000,00 \mathrm{~b}$ & $3776,67 \mathrm{~d}$ \\
BRS 1010 & $88,93 \mathrm{a}$ & $12,20 \mathrm{a}$ & $359333,33 \mathrm{~b}$ & $3692,33 \mathrm{~d}$ \\
BRS 388 & $78,70 \mathrm{~b}$ & $10,77 \mathrm{a}$ & $286333,33 \mathrm{~b}$ & $3648,33 \mathrm{~d}$ \\
BRS 7380 & $93,10 \mathrm{a}$ & $12,93 \mathrm{a}$ & $226666,67 \mathrm{~b}$ & $3528,33 \mathrm{~d}$ \\
TMG 7063 & $79,37 \mathrm{~b}$ & $11,13 \mathrm{a}$ & $294666,67 \mathrm{~b}$ & $3326,00 \mathrm{e}$ \\
SYN 13610 & $90,20 \mathrm{a}$ & $13,93 \mathrm{a}$ & $427000,00 \mathrm{a}$ & $3255,00 \mathrm{e}$ \\
BMX 63 I 64 & $88,10 \mathrm{a}$ & $19,43 \mathrm{a}$ & $320000,00 \mathrm{~b}$ & $3200,67 \mathrm{e}$ \\
AS 3730 & $91,70 \mathrm{a}$ & $15,77 \mathrm{a}$ & $386333,33 \mathrm{a}$ & $3176,67 \mathrm{e}$ \\
RK 7518 & $85,70 \mathrm{a}$ & $17,20 \mathrm{a}$ & $466444,33 \mathrm{a}$ & $3138,00 \mathrm{e}$ \\
BRS 413 & $69,43 \mathrm{~b}$ & $9,87 \mathrm{a}$ & $321777,67 \mathrm{~b}$ & $2992,67 \mathrm{f}$ \\
M 5947 & $79,27 \mathrm{~b}$ & $11,37 \mathrm{a}$ & $286000,00 \mathrm{~b}$ & $2984,67 \mathrm{f}$ \\
RK 6316 & $67,87 \mathrm{~b}$ & $14,00 \mathrm{a}$ & $482666,67 \mathrm{a}$ & $2937,00 \mathrm{f}$ \\
RK 6813 & $78,43 \mathrm{~b}$ & $14,43 \mathrm{a}$ & $414666,67 \mathrm{a}$ & $2890,67 \mathrm{f}$ \\
NS 6990 & $72,00 \mathrm{~b}$ & $8,67 \mathrm{a}$ & $307333,33 \mathrm{~b}$ & $2780,33 \mathrm{f}$ \\
SYN 1366 & $84,77 \mathrm{a}$ & $13,77 \mathrm{a}$ & $400889,00 \mathrm{a}$ & $2666,00 \mathrm{f}$ \\
M 5917 & $77,93 \mathrm{~b}$ & $11,80 \mathrm{a}$ & $302222,33 \mathrm{~b}$ & $2416,33 \mathrm{~g}$ \\
BRS 1003 & $76,43 \mathrm{~b}$ & $12,37 \mathrm{a}$ & $294666,67 \mathrm{~b}$ & $2412,67 \mathrm{~g}$ \\
M 6210 & $80,63 \mathrm{~b}$ & $13,37 \mathrm{a}$ & $342666,67 \mathrm{~b}$ & $2249,33 \mathrm{~g}$ \\
M 6410 & $79,20 \mathrm{~b}$ & $11,13 \mathrm{a}$ & $375666,67 \mathrm{a}$ & $2241,00 \mathrm{~g}$ \\
FTR 4160 & $81,27 \mathrm{~b}$ & $11,37 \mathrm{a}$ & $420666,67 \mathrm{a}$ & $2055,67 \mathrm{~h}$ \\
BRS 433 & $73,70 \mathrm{~b}$ & $12,27 \mathrm{a}$ & $340666,67 \mathrm{~b}$ & $1986,00 \mathrm{~h}$ \\
BRS 399 & $68,87 \mathrm{~b}$ & $9,60 \mathrm{a}$ & $260666,67 \mathrm{~b}$ & $1945,67 \mathrm{~h}$ \\
SYN 15640 & $87,10 \mathrm{a}$ & $13,53 \mathrm{a}$ & $498555,67 \mathrm{a}$ & $1592,00 \mathrm{~h}$ \\
\hline Média & 82,02 & 13,08 & 358055,56 & 3209,72 \\
\hline CV\% & 9,06 & 24,24 & 21,27 & 7,77 \\
\hline F & $2,89 * *$ & $2,04 *$ & $2,95 * *$ & $40,91 * *$ \\
\hline
\end{tabular}

Médias de três repetições seguidas de mesmas letras minúsculas na coluna não diferem estatisticamente pelo teste de Scott Knott a 5\% de probabilidade. APM = altura de planta na maturação em $\mathrm{cm}$; AIV = altura de inserção da primeira vagem em $\mathrm{cm} ; \mathrm{POP}=$ população de plantas em plantas ha ${ }^{-1} \mathrm{e} \mathrm{PG}=$ produtividade de grãos em $\mathrm{kg} \mathrm{ha}^{-1}$.

Tabela 3. Média dos caracteres agronômicos obtidos de ensaio comparativo de 28 cultivares de soja, conduzido em Mococa, SP. Safra 2018/19.

(Continua)

\begin{tabular}{ccccc}
\hline Cultivar & APM & AIV & POP & PG \\
\hline AS 3590 & $53,50 \mathrm{a}$ & $9,58 \mathrm{a}$ & $300000,00 \mathrm{e}$ & $2916,67 \mathrm{a}$ \\
AS 3680 & $60,83 \mathrm{a}$ & $10,25 \mathrm{a}$ & $330000,00 \mathrm{~d}$ & $2766,67 \mathrm{a}$ \\
RK 6316 & $58,17 \mathrm{a}$ & $12,50 \mathrm{a}$ & $423333,33 \mathrm{~b}$ & $2533,33 \mathrm{~b}$ \\
M 6210 & $56,75 \mathrm{a}$ & $12,83 \mathrm{a}$ & $290000,00 \mathrm{e}$ & $2233,33 \mathrm{c}$ \\
RK 7518 & $67,50 \mathrm{a}$ & $14,67 \mathrm{a}$ & $290000,00 \mathrm{e}$ & $2166,67 \mathrm{c}$
\end{tabular}


Tabela 3. Média dos caracteres agronômicos obtidos de ensaio comparativo de 28 cultivares de soja, conduzido em Mococa, SP. Safra 2018/19.

(Conclusão)

\begin{tabular}{ccccc}
\hline Cultivar & APM & AIV & POP & PG \\
TMG 7067 & $49,75 \mathrm{a}$ & $10,08 \mathrm{a}$ & $296666,67 \mathrm{e}$ & $2166,67 \mathrm{c}$ \\
AS 3730 & $62,00 \mathrm{a}$ & $10,33 \mathrm{a}$ & $310000,00 \mathrm{~d}$ & $2066,67 \mathrm{~d}$ \\
NS 6906 & $45,50 \mathrm{a}$ & $7,58 \mathrm{a}$ & $370000,00 \mathrm{c}$ & $2016,67 \mathrm{~d}$ \\
LG 162 & $48,33 \mathrm{a}$ & $10,25 \mathrm{a}$ & $366666,67 \mathrm{c}$ & $2000,00 \mathrm{~d}$ \\
LG 158 & $52,42 \mathrm{a}$ & $10,92 \mathrm{a}$ & $463333,33 \mathrm{a}$ & $1933,33 \mathrm{~d}$ \\
LG 179 & $54,08 \mathrm{a}$ & $10,42 \mathrm{a}$ & $320000,00 \mathrm{~d}$ & $1916,67 \mathrm{~d}$ \\
RK 6813 & $49,42 \mathrm{a}$ & $8,75 \mathrm{a}$ & $410000,00 \mathrm{c}$ & $1850,00 \mathrm{~d}$ \\
SYN 1366 & $55,75 \mathrm{a}$ & $6,33 \mathrm{a}$ & $403333,33 \mathrm{c}$ & $1833,33 \mathrm{~d}$ \\
TMG 7062 & $54,50 \mathrm{a}$ & $10,58 \mathrm{a}$ & $320000,00 \mathrm{~d}$ & $1783,33 \mathrm{~d}$ \\
BRS 7380 & $55,25 \mathrm{a}$ & $12,17 \mathrm{a}$ & $346666,67 \mathrm{~d}$ & $1766,67 \mathrm{~d}$ \\
SYN 15640 & $39,08 \mathrm{a}$ & $6,33 \mathrm{a}$ & $293333,33 \mathrm{e}$ & $1750,00 \mathrm{~d}$ \\
M 5947 & $54,83 \mathrm{a}$ & $8,25 \mathrm{a}$ & $273333,33 \mathrm{e}$ & $1716,67 \mathrm{e}$ \\
BRS 388 & $52,00 \mathrm{a}$ & $11,42 \mathrm{a}$ & $336666,67 \mathrm{~d}$ & $1700,00 \mathrm{e}$ \\
SYN 13610 & $52,42 \mathrm{a}$ & $10,58 \mathrm{a}$ & $236666,67 \mathrm{f}$ & $1700,00 \mathrm{e}$ \\
TMG 7063 & $51,92 \mathrm{a}$ & $9,33 \mathrm{a}$ & $400000,00 \mathrm{c}$ & $1683,33 \mathrm{e}$ \\
BRS 1001 & $59,17 \mathrm{a}$ & $10,25 \mathrm{a}$ & $273333,33 \mathrm{e}$ & $1600,00 \mathrm{e}$ \\
NS 6700 & $47,42 \mathrm{a}$ & $9,67 \mathrm{a}$ & $343333,33 \mathrm{~d}$ & $1600,00 \mathrm{e}$ \\
M 6410 & $63,25 \mathrm{a}$ & $11,83 \mathrm{a}$ & $386666,67 \mathrm{c}$ & $1583,33 \mathrm{e}$ \\
M 5917 & $51,75 \mathrm{a}$ & $10,67 \mathrm{a}$ & $426666,67 \mathrm{~b}$ & $1550,00 \mathrm{e}$ \\
NS 6990 & $53,50 \mathrm{a}$ & $9,92 \mathrm{a}$ & $340000,00 \mathrm{~d}$ & $1533,33 \mathrm{e}$ \\
BRS 1074 & $52,58 \mathrm{a}$ & $12,25 \mathrm{a}$ & $340000,00 \mathrm{~d}$ & $1533,33 \mathrm{e}$ \\
AS 3610 & $64,17 \mathrm{a}$ & $10,58 \mathrm{a}$ & $383333,33 \mathrm{c}$ & $1433,33 \mathrm{e}$ \\
VALIOSA & $74,75 \mathrm{a}$ & $9,17 \mathrm{a}$ & $330000,00 \mathrm{~d}$ & $1283,33 \mathrm{e}$ \\
\hline Média & 55,02 & 10,27 & 342976,19 & 1879,17 \\
\hline CV\% & 20,28 & 24,00 & 6,30 & 9,65 \\
\hline F & $1,22 N S$ & $1,66 N S$ & $18,99 * *$ & $13,42 * *$ \\
\hline
\end{tabular}

Médias de três repetições seguidas de mesmas letras minúsculas na coluna não diferem estatisticamente pelo teste de Scott Knott a 5\% de probabilidade. APM = altura de planta na maturação em $\mathrm{cm}$; AIV = altura de inserção da primeira vagem em $\mathrm{cm}$; $\mathrm{POP}=$ população de plantas em plantas ha $\mathrm{h}^{-1} \mathrm{e} \mathrm{PG}=$ produtividade de grãos em $\mathrm{kg} \mathrm{ha}^{-1}$.

Tabela 4. Média dos caracteres agronômicos obtidos de ensaio comparativo de 29 cultivares de soja, conduzido em Mogi Mirim, SP. Safra 2018/19.

(Continua)

\begin{tabular}{ccccc}
\hline Cultivar & APM & AIV & POP & PG \\
\hline RK 6316 & $71,33 \mathrm{c}$ & $18,33 \mathrm{a}$ & $295333,33 \mathrm{a}$ & $5050,33 \mathrm{a}$ \\
AS 3730 & $96,67 \mathrm{~b}$ & $20,67 \mathrm{a}$ & $204000,00 \mathrm{c}$ & $4867,00 \mathrm{a}$ \\
AS 3590 & $88,00 \mathrm{c}$ & $19,67 \mathrm{a}$ & $186000,00 \mathrm{~d}$ & $4710,33 \mathrm{~b}$ \\
RK 7518 & $99,00 \mathrm{~b}$ & $24,67 \mathrm{a}$ & $217333,33 \mathrm{c}$ & $4548,67 \mathrm{~b}$ \\
SYN 15640 & $82,00 \mathrm{c}$ & $11,33 \mathrm{c}$ & $190666,67 \mathrm{~d}$ & $4382,00 \mathrm{c}$
\end{tabular}


Tabela 4. Média dos caracteres agronômicos obtidos de ensaio comparativo de 29 cultivares de soja, conduzido em Mogi Mirim, SP. Safra 2018/19.

(Conclusão)

\begin{tabular}{ccccc}
\hline Cultivar & APM & AIV & POP & PG \\
TMG 7063 & $96,67 \mathrm{~b}$ & $21,00 \mathrm{a}$ & $215333,33 \mathrm{c}$ & $4356,67 \mathrm{c}$ \\
BRS 1001 & $93,33 \mathrm{~b}$ & $21,00 \mathrm{a}$ & $205333,33 \mathrm{c}$ & $4230,67 \mathrm{c}$ \\
BMX 63 I 64 & $91,67 \mathrm{c}$ & $20,33 \mathrm{a}$ & $230000,00 \mathrm{c}$ & $4220,33 \mathrm{c}$ \\
BS 2606 & $83,00 \mathrm{c}$ & $16,33 \mathrm{~b}$ & $226666,67 \mathrm{c}$ & $4149,00 \mathrm{c}$ \\
NS 6906 & $87,33 \mathrm{c}$ & $18,33 \mathrm{a}$ & $174666,67 \mathrm{~d}$ & $4140,00 \mathrm{c}$ \\
M 6210 & $90,33 \mathrm{c}$ & $18,33 \mathrm{a}$ & $240666,67 \mathrm{~b}$ & $4046,33 \mathrm{c}$ \\
AS 3680 & $96,00 \mathrm{~b}$ & $16,67 \mathrm{~b}$ & $180666,67 \mathrm{~d}$ & $4034,67 \mathrm{c}$ \\
SYN 1366 & $83,33 \mathrm{c}$ & $10,00 \mathrm{c}$ & $176000,00 \mathrm{~d}$ & $4007,33 \mathrm{c}$ \\
SYN 13610 & $106,67 \mathrm{a}$ & $22,33 \mathrm{a}$ & $192666,67 \mathrm{~d}$ & $3976,00 \mathrm{c}$ \\
BRS 1003 & $89,67 \mathrm{c}$ & $22,00 \mathrm{a}$ & $244000,00 \mathrm{~b}$ & $3876,67 \mathrm{~d}$ \\
BRS 7380 & $114,67 \mathrm{a}$ & $23,33 \mathrm{a}$ & $170000,00 \mathrm{~d}$ & $3770,67 \mathrm{~d}$ \\
BRS 413 & $75,00 \mathrm{c}$ & $18,33 \mathrm{a}$ & $246000,00 \mathrm{~b}$ & $3727,67 \mathrm{~d}$ \\
M 5917 & $85,33 \mathrm{c}$ & $17,67 \mathrm{~b}$ & $186000,00 \mathrm{~d}$ & $3664,33 \mathrm{~d}$ \\
M 6410 & $87,00 \mathrm{c}$ & $16,67 \mathrm{~b}$ & $205333,33 \mathrm{c}$ & $3654,00 \mathrm{~d}$ \\
NS 6700 & $81,67 \mathrm{c}$ & $16,00 \mathrm{~b}$ & $165333,33 \mathrm{~d}$ & $3632,67 \mathrm{~d}$ \\
VALIOSA & $110,33 \mathrm{a}$ & $21,33 \mathrm{a}$ & $180000,00 \mathrm{~d}$ & $3579,00 \mathrm{~d}$ \\
FTR 4160 & $78,33 \mathrm{c}$ & $14,67 \mathrm{~b}$ & $251333,33 \mathrm{~b}$ & $3548,00 \mathrm{~d}$ \\
M 5947 & $81,00 \mathrm{c}$ & $15,67 \mathrm{~b}$ & $212000,00 \mathrm{c}$ & $3476,33 \mathrm{~d}$ \\
NS 6990 & $81,67 \mathrm{c}$ & $15,33 \mathrm{~b}$ & $107333,33 \mathrm{f}$ & $3475,33 \mathrm{~d}$ \\
RK 6813 & $88,33 \mathrm{c}$ & $19,00 \mathrm{a}$ & $183333,33 \mathrm{~d}$ & $3405,00 \mathrm{e}$ \\
BRS 399 & $80,00 \mathrm{c}$ & $12,33 \mathrm{c}$ & $166666,67 \mathrm{~d}$ & $3338,00 \mathrm{e}$ \\
BRS 1010 & $102,50 \mathrm{~b}$ & $14,00 \mathrm{~b}$ & $186333,33 \mathrm{~d}$ & $3181,33 \mathrm{e}$ \\
BRS 388 & $85,33 \mathrm{c}$ & $17,33 \mathrm{~b}$ & $185333,33 \mathrm{~d}$ & $3174,67 \mathrm{e}$ \\
TMG 7062 & $84,00 \mathrm{c}$ & $17,50 \mathrm{~b}$ & $138333,33 \mathrm{e}$ & $2962,67 \mathrm{e}$ \\
\hline Média & 89,32 & 17,96 & 198712,64 & 3902,95 \\
\hline CV\% & 7,01 & 16,41 & 7,09 & 4,79 \\
\hline F & $8,06 * *$ & $4,31 * *$ & $20,79 * *$ & $22,86 * *$ \\
\hline
\end{tabular}

Médias de três repetições seguidas de mesmas letras minúsculas na coluna não diferem estatisticamente pelo teste de Scott Knott a $5 \%$ de probabilidade. APM = altura de planta na maturação em $\mathrm{cm}$; AIV = altura de inserção da primeira vagem em $\mathrm{cm}$; $\mathrm{POP}=$ população de plantas em plantas $\mathrm{ha}^{-1} \mathrm{e} \mathrm{PG}=$ produtividade de grãos em $\mathrm{kg} \mathrm{ha}^{-1}$.

Tabela 5. Média dos caracteres agronômicos obtidos de ensaio comparativo de 35 cultivares de soja, conduzido em Tanabi, SP. Safra 2018/19.

(Continua)

\begin{tabular}{ccccc}
\hline Cultivar & APM & AIV & POP & PG \\
\hline SYN 15640 & $79,75 \mathrm{~b}$ & $37,75 \mathrm{a}$ & $298889,00 \mathrm{~b}$ & $2894,00 \mathrm{a}$ \\
P96 R70 & $86,83 \mathrm{a}$ & $21,50 \mathrm{c}$ & $303333,33 \mathrm{~b}$ & $2853,00 \mathrm{a}$ \\
VALIOSA & $71,30 \mathrm{c}$ & $15,63 \mathrm{~d}$ & $370666,67 \mathrm{a}$ & $2450,00 \mathrm{~b}$ \\
M 7739 & $89,75 \mathrm{a}$ & $21,08 \mathrm{c}$ & $337777,67 \mathrm{a}$ & $2307,33 \mathrm{~b}$ \\
TMG 7063 & $81,26 \mathrm{~b}$ & $16,70 \mathrm{~d}$ & $332000,00 \mathrm{a}$ & $2286,00 \mathrm{~b}$
\end{tabular}


Tabela 5. Média dos caracteres agronômicos obtidos de ensaio comparativo de 35 cultivares de soja, conduzido em Tanabi, SP. Safra 2018/19.

(Conclusão)

\begin{tabular}{|c|c|c|c|c|}
\hline Cultivar & APM & AIV & POP & PG \\
\hline M 6410 & $66,00 \mathrm{c}$ & $18,91 \mathrm{c}$ & 333333,33 a & $2225,33 \mathrm{~b}$ \\
\hline TMG 7067 & $74,41 \mathrm{c}$ & $18,33 \mathrm{c}$ & $322222,00 \mathrm{a}$ & $2208,00 \mathrm{~b}$ \\
\hline P97 R22 & 90,08 a & $26,91 \mathrm{~b}$ & $268889,00 \mathrm{~b}$ & $2183,33 \mathrm{~b}$ \\
\hline P97 R50 & $102,5 \mathrm{a}$ & $25,66 \mathrm{~b}$ & $282222,33 \mathrm{~b}$ & $2163,33 \mathrm{~b}$ \\
\hline M 7110 & $51,25 \mathrm{~d}$ & $10,58 \mathrm{e}$ & 323333,33 a & $2152,33 \mathrm{~b}$ \\
\hline PARANAIBA & $95,00 \mathrm{a}$ & $25,00 \mathrm{~b}$ & 297777,67 b & $2056,33 \mathrm{~b}$ \\
\hline NA 7337 & $75,75 \mathrm{~b}$ & $21,58 \mathrm{c}$ & $303333,33 \mathrm{~b}$ & 2030,66 c \\
\hline TMG 7061 & $78,00 \mathrm{~b}$ & $17,91 \mathrm{c}$ & 318889,00 a & 2005,66 c \\
\hline FTR ANTARI & $57,93 \mathrm{~d}$ & $10,80 \mathrm{e}$ & 341333,33 a & $1985,66 \mathrm{c}$ \\
\hline DM $68 i 69$ & $58,91 \mathrm{~d}$ & $16,58 \mathrm{~d}$ & 356666,67 a & $1956,66 \mathrm{c}$ \\
\hline NA 5909 & $91,91 \mathrm{a}$ & $20,08 \mathrm{c}$ & $238889,00 \mathrm{~b}$ & $1904,33 \mathrm{c}$ \\
\hline NS 7007 & 96,66 a & $24,08 \mathrm{~b}$ & $258888,67 \mathrm{~b}$ & $1876,33 \mathrm{c}$ \\
\hline LG 79 & $66,58 \mathrm{c}$ & $16,66 \mathrm{~d}$ & $298889,00 \mathrm{~b}$ & $1788,66 \mathrm{~d}$ \\
\hline TMG 7062 & $65,25 \mathrm{c}$ & $18,91 \mathrm{c}$ & $270000,00 \mathrm{~b}$ & $1716,66 \mathrm{~d}$ \\
\hline LG 54 & $66,33 \mathrm{c}$ & $16,08 \mathrm{~d}$ & 313333,33 a & $1696,66 \mathrm{~d}$ \\
\hline P9 R10 & $79,83 \mathrm{~b}$ & $18,66 \mathrm{c}$ & $293333,33 \mathrm{~b}$ & $1679,00 \mathrm{~d}$ \\
\hline AS 3730 & $71,50 \mathrm{c}$ & $15,75 \mathrm{~d}$ & $280000,00 \mathrm{~b}$ & $1624,00 \mathrm{~d}$ \\
\hline P96 R20 & $52,83 \mathrm{~d}$ & $11,91 \mathrm{e}$ & 313333,33 a & $1622,33 \mathrm{~d}$ \\
\hline BMX DESAFIO & $80,91 \mathrm{~b}$ & $17,91 \mathrm{c}$ & $292222,33 \mathrm{~b}$ & $1606,00 \mathrm{~d}$ \\
\hline LG 11 & $72,33 \mathrm{c}$ & $24,25 \mathrm{~b}$ & 284444,67 b & $1569,66 \mathrm{~d}$ \\
\hline P95 R 90 & $80,33 \mathrm{~b}$ & $20,58 \mathrm{c}$ & 294444,33 b & $1528,66 \mathrm{~d}$ \\
\hline NS 7202 & $70,58 \mathrm{c}$ & $16,00 \mathrm{~d}$ & $278889,00 \mathrm{~b}$ & $1522,66 \mathrm{~d}$ \\
\hline BMX 7166 & $80,66 \mathrm{~b}$ & $17,75 \mathrm{c}$ & $263333,67 \mathrm{~b}$ & $1498,00 \mathrm{~d}$ \\
\hline BMX FOCO & $77,41 \mathrm{~b}$ & $15,83 \mathrm{~d}$ & $238889,00 \mathrm{~b}$ & $1490,66 \mathrm{~d}$ \\
\hline CD 2700 & $71,25 \mathrm{c}$ & $16,50 \mathrm{~d}$ & $312222,00 \mathrm{a}$ & $1474,00 \mathrm{~d}$ \\
\hline SYN 13610 & $80,91 \mathrm{~b}$ & $15,16 \mathrm{~d}$ & $292222,33 \mathrm{~b}$ & $1427,33 \mathrm{e}$ \\
\hline BRS 7380 & $81,00 \mathrm{~b}$ & $17,91 \mathrm{c}$ & $271111,00 \mathrm{~b}$ & $1349,66 \mathrm{e}$ \\
\hline LG 19 & 88,41 a & $17,75 \mathrm{c}$ & $275555,67 \mathrm{~b}$ & $1343,66 \mathrm{e}$ \\
\hline BMX ÚNICA & $61,25 \mathrm{~d}$ & $14,83 \mathrm{~d}$ & $303333,67 \mathrm{~b}$ & $1208,66 \mathrm{f}$ \\
\hline LG 60i63 & $71,91 \mathrm{c}$ & $17,58 \mathrm{c}$ & $260000,00 \mathrm{~b}$ & $1127,00 \mathrm{f}$ \\
\hline Média & 76,19 & 18,83 & 297733,35 & 1851,81 \\
\hline $\mathrm{CV} \%$ & 8,68 & 15,12 & 10,34 & 6,85 \\
\hline $\mathrm{F}$ & $10,26^{* *}$ & $9,46^{* *}$ & $3,00 * *$ & $33,42 * *$ \\
\hline
\end{tabular}

Médias de três repetições seguidas de mesmas letras minúsculas na coluna não diferem estatisticamente pelo teste de Scott Knott a 5\% de probabilidade. APM = altura de planta na maturação em $\mathrm{cm}$; AIV = altura de inserção da primeira vagem em $\mathrm{cm} ; \mathrm{POP}=$ população de plantas em plantas ha ${ }^{-1}$ e $\mathrm{PG}=$ produtividade de grãos em $\mathrm{kg} \mathrm{ha}^{-1}$. 
Devem-se considerar as condições onde foram realizados os experimentos, pois, a produtividade da cultura é definida pela interação entre planta, ambiente de produção e manejo (MAUAD et al., 2010).

\section{CONCLUSÃO}

Existem cultivares comerciais que demonstram tolerância às condições adversas de cada região apresentando produtividades superiores à média do estado de São Paulo.

A maioria das cultivares apresenta altura de plantas e de inserção da primeira vagem adequadas à colheita mecanizada, com valores acima de $50 \mathrm{~cm}$ e $12 \mathrm{~cm}$, respectivamente.

É necessária a continuação dos estudos para melhor recomendação das cultivares visando atender a demanda de produtores por informações imparciais na escolha de cultivares mais adequadas ao plantio regionalizado.

\section{REFERÊNCIAS}

BONETTI, L. P. Cultivares e seu melhoramento. In: VERNETTI, F.J. (Ed.). Soja: genética e melhoramento. Campinas: Fundação Cargill, p.741-94, 1993.

BORGES, W. L. B.et al. Avaliação regional de cultivares de soja no estado de São Paulo - safra 2017/18. Nucleus, Edição Especial 2018. $3^{\circ}$ Encontro Técnico sobre as culturas da soja e milho no Noroeste Paulista. p. 73-90. 2018. Disponível em: http://dx.doi.org/10.3738/1982.2278.3006

CONAB - Companhia Nacional de Abastecimento Agrícola - (2019) Quarto levantamento de grãos. Safra 2018/19. Acesso em: 10/01/2019. Disponível em: <https://www.conab.gov.br/info-agro/ safras/ graos>.

CRUZ, T. V.et al.Componentes de produção de soja em diferentes épocas de semeadura, no Oeste da Bahia. Bioscience Journal, v. 26, n. 5, p. 709-716, 2010. Disponível em: http://www.seer.ufu.br/index.php/biosciencejournal/article/viewFile/7198/525

EVANS, L. T. Crop evolution, adaptation and yield. Cambridge University, Cambridge, 1993. 500 p.

YUYAMA, K. Avaliação de algumas características agronômicas e morfofisiológicas de cinco cultivares de soja (Glycine max (L.) Merrill), cultivados em solo de várzea e de terra firme da Amazônia Central. Tese (Doutorado em Agronomia - Produção Vegetal) - Universidade Estadual Paulista, Jaboticabal,1991. 130f

MAUAD, M. et al. Influência da densidade de semeadura sobre características agronômicas na cultura da soja. Agrarian, v. 3, n. 9, p. 175-181, 2010. Disponível em:

http://ojs.ufgd.edu.br/index.php/agrarian/article/view/75

PEIXOTO, C. P. et al. Épocas de semeadura e densidades de plantas de soja: I. Componentes da produção e rendimento de grãos. Scientia Agrícola, Piracicaba, v. 57, n. 1, p. 89-96, 2000. Disponível em: http://dx.doi.org/10.1590/S0103-90162000000100015

REZENDE, P. M.; CARVALHO, E. A. Avaliação de cultivares de soja (Glycine max (L.) Merrill) para o Sul de Minas. Disponível em: http://www.scielo.br/pdf/cagro/v31n6/a03v31n6

USDA - UNITAD STATES DEPARTAMENT OF AGRICULTURE (2018) Disponível em: 〈http://usdamannlibcornelledu/usda/current/worldag-production/worldag-production-09-12-2018pdf > Acesso em: 11/09/2018. 